\title{
GCU
}

Glasgow Caledonian

University

University for the Common Good

\section{Conservative management of pelvic organ prolapse}

Hagen, Suzanne; Thakar, Ranee

Published in:

Obstetrics, Gynaecology and Reproductive Medicine

DOI:

10.1016/j.ogrm.2012.02.003

Publication date:

2012

Document Version

Author accepted manuscript

Link to publication in ResearchOnline

Citation for published version (Harvard):

Hagen, S \& Thakar, R 2012, 'Conservative management of pelvic organ prolapse', Obstetrics, Gynaecology and Reproductive Medicine, vol. 22, no. 5, pp. 118-122. https://doi.org/10.1016/j.ogrm.2012.02.003

\section{General rights}

Copyright and moral rights for the publications made accessible in the public portal are retained by the authors and/or other copyright owners and it is a condition of accessing publications that users recognise and abide by the legal requirements associated with these rights.

Take down policy

If you believe that this document breaches copyright please view our takedown policy at https://edshare.gcu.ac.uk/id/eprint/5179 for details of how to contact us. 


\section{TITLE PAGE}

Title: $\quad$ Conservative management of pelvic organ prolapse

\section{Authors:}

Professor Suzanne Hagen, PhD CStat CSci

Nursing, Midwifery and Allied Health Professions Research Unit

Glasgow Caledonian University

Cowcaddens Road

Glasgow G4 0BA

Tel $01413318104 /$ fax 01413318101

Email: s.hagen@gcu.ac.uk

Miss Ranee Thakar MD, MRCOG

Consultant Urogynaecologist

Croydon University Hospital

Croydon

CR7 7YE

Tel 02084013154

Email: raneethakar@yahoo.co.uk 


\section{ABSTRACT}

Pelvic organ prolapse (POP) is a common female problem which is defined as the symptomatic descent of the walls or apex of the vagina. Symptoms of a vaginal bulge or protrusion, pelvic heaviness, bladder, bowel and sexual dysfunction are often associated with the problem. Treatments include surgery and conservative management. Conservative approaches to the treatment of prolapse are often used in cases of mild to moderate prolapse; for those who wish to have more children; the frail or those unwilling to undergo surgery; and those awaiting surgery. This article describes the conservative methods available to women presenting with pelvic organ prolapse including lifestyle advice, pelvic floor muscle training and vaginal pessaries. (111 words)

\section{KEYWORDS (MESH index)}

Pelvic organ prolapse, conservative management, lifestyle advice, pelvic floor muscle training, pessary 


\section{MAIN TEXT}

\section{Introduction}

Pelvic organ prolapse is a common female condition. Around $50 \%$ of parous women have prolapse on examination (anatomical prolapse). The prevalence of prolapse accompanied by bothersome symptoms (symptomatic prolapse) is lower; reported to be around $3 \%$ in one US survey by Nygaard and colleagues. Women with prolapse can experience a variety of pelvic floor symptoms. Treatments include surgery and conservative management. Choice of treatment depends on the severity of the prolapse and its symptoms, and the woman's general health and preferences. Conservative treatment is generally considered for women with a mild degree of prolapse, those who wish to have more children, the frail or those unwilling to undergo surgery. Conservative approaches can also be used prior to surgery, for example as an adjunct, or as an interim measure. Conservative treatment is defined here as lifestyle interventions, physical therapies (pelvic floor muscle training) and pessaries.

The benefits of conservative therapies are that they tend to be inexpensive, have low risk of adverse effects and do not prejudice the use of subsequent treatments such as surgery. As the prevalence of POP is high, and healthcare budgets are constrained, conservative treatment is the main form of management in primary care.

The aims of conservative treatment in the management of POP include: 
- to prevent the prolapse becoming worse;

- to help decrease the frequency or severity of symptoms caused by prolapse (vaginal bulge/protrusion, pelvic heaviness, vaginal symptoms, backache, urinary, bowel and sexual symptoms);

- to avert or delay the need for surgery.

To date, only a relatively small number of intervention studies of adequate size have been carried out to assess the effectiveness of conservative treatments for POP.

The aim of this article is to describe three conservative approaches to POP which can be considered for women presenting with this problem: lifestyle advice, pelvic floor muscle training and vaginal pessaries.

\section{Lifestyle advice}

Lifestyle interventions include weight loss, reducing exacerbating activities (e.g. heavy lifting, coughing) and treating constipation. These interventions seek to avoid exacerbation of the prolapse by decreasing intra-abdominal pressure.

\section{Effectiveness of lifestyle advice}

The extent to which any of these lifestyle changes are effective in managing prolapse is unknown as there have been no randomised controlled trials of lifestyle interventions in this population. Occupational heavy lifting has been found in several observational studies to be associated with symptomatic POP. Although there are 
no controlled trials to indicate definitively the effect on POP of interventions to alter lifting behaviour, avoidance of heavy lifting or methods of correct lifting technique should be encouraged in women presenting with POP symptoms. Observational studies have consistently found an association between being overweight and POP symptoms. This would tend to suggest that weight management may have a role to play in preventing and possibly treating POP. Randomised controlled trials of weight management interventions are however needed to evaluate this hypothesis. Studies have also reported an association between constipation and symptoms of prolapse but there is no evidence to support a causal relationship: prolapse, particularly prolapse of the posterior vaginal wall, may lead to problems with evacuation, but conversely constipation can cause straining which contributes to the development of prolapse. It is tentatively suggested therefore that women with POP symptoms should be advised to avoid constipation. Interestingly the studies assessing lifestyle factors and their association with prolapse have often found an association with the symptoms of prolapse reported, but failed to find an association with POP severity as measured on examination. This lack of correlation between the presence (or extent) of prolapse symptoms and the anatomical severity has been noted by several authors in the literature. For example, some women with prolapse extending beyond the hymen may report no symptoms, whilst others with mild prolapse have very bothersome symptoms. This highlights the importance of recording both symptoms and severity of prolapse in research and clinical practice.

\section{Recommending lifestyle advice}

Advice on appropriate lifestyle changes (see box 1) can be given to any woman with prolapse to help reduce intra-abdominal pressure to ease the downward pressure on 
the prolapsed area. The advice can also help with other co-existing bladder or bowel symptoms. For example: avoiding constipation via diet and fluid adjustments or use of medication; altering lifting habits (e.g. lift multiple small weights rather than single large weights); altering exercise from high impact (e.g. running) to low or no impact (e.g. swimming); and losing weight. Specific advice to help with urinary symptoms includes timed regular voiding, bladder training, double voiding without straining and reducing bladder irritants such as caffeine. Advice to avoid straining to defaecate may include diet, fluid intake advice and advice on correcting defaecation technique.

Any changes which can be made over a short or long-term period to reduce prolapse and related symptoms should be advocated. It should be stressed to the woman that even if surgical management is selected, this lifestyle advice must be adhered to post-operatively to enhance long-term success.

\section{Box 1. Lifestyle Advice}

- Avoid constipation

- Weight loss

- Avoid heavy lifting

- avoid high impact exercise

- Smoking cessation

\section{Pelvic Floor Muscle Training}

The pelvic floor muscles play a critical role in giving structural support to the pelvic organs and pelvic openings (Figure 1). Pelvic floor muscle activity adjusts to 
variations in posture and intra-abdominal pressure. It is hypothesised that improving pelvic floor muscle function (strength, endurance and coordination) may improve this structural support for the pelvic organs.

Figure 1. The female pelvic floor muscles

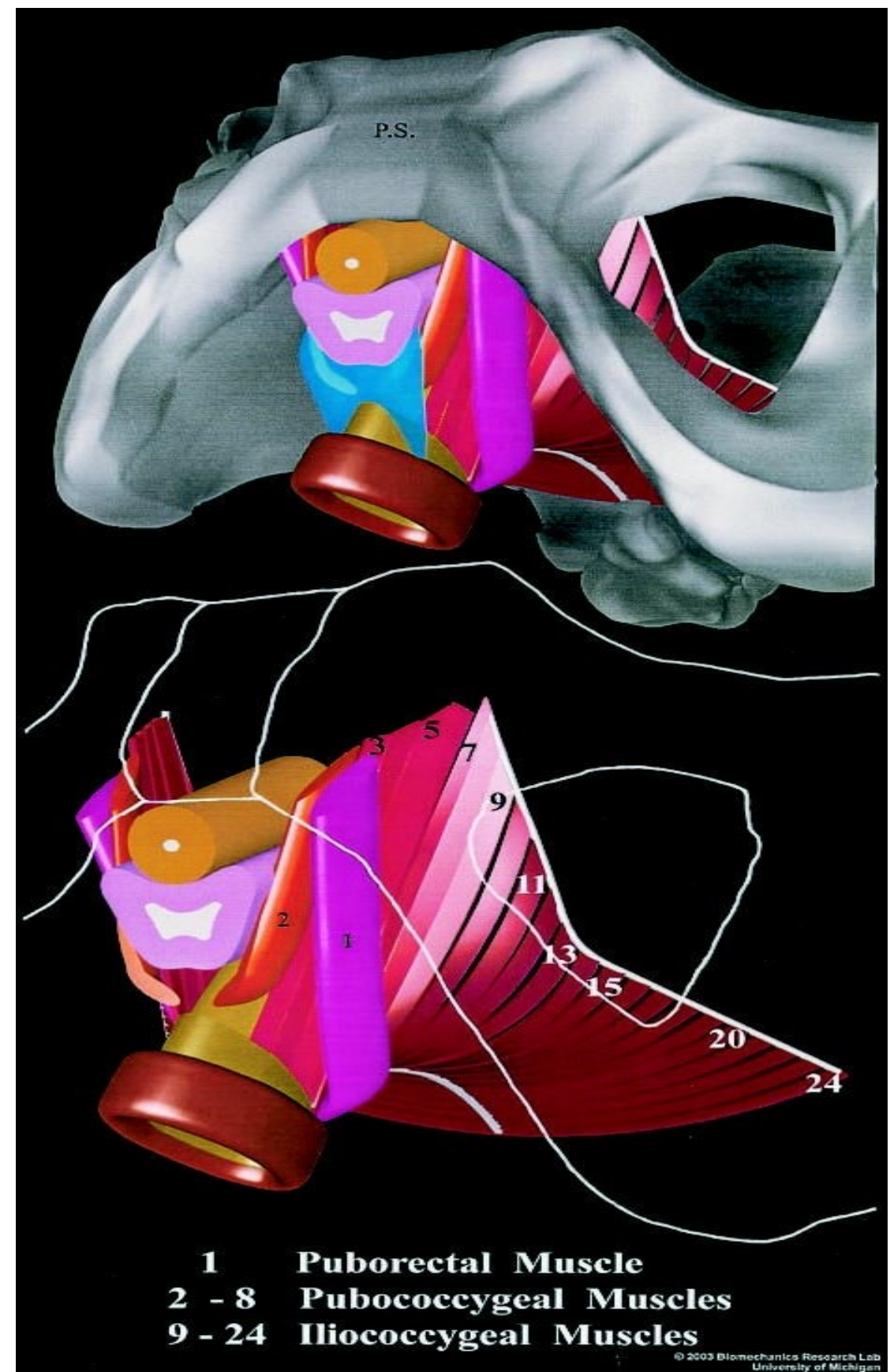

There are two main hypotheses for pelvic floor muscle training (PFMT) being a potentially effective treatment for POP. Firstly, intensive strength training for the 
pelvic floor muscles may build up pelvic structural support by increasing muscle volume and elevating the levator plate (pelvic floor muscles and pelvic organs) to a higher position inside the pelvis. This increased strength may hypertrophy and improve stiffness of the pelvic floor muscles. DeLancey demonstrated that women with POP generated less vaginal closure force during a maximal voluntary contraction than controls. Improving pelvic floor muscle strength in women with POP may have an important role to play in the treatment of POP.

Secondly, an intentional, effective pelvic floor muscle contraction prior to and during effort such as a cough or sneeze (known as the Knack) has been shown to reduce leakage from stress urinary incontinence, thus this "pre-contraction" has become a standard element of PFMT for urinary incontinence. There are no studies looking at the effectiveness of the Knack for POP. Some authors have recommended precontracting the pelvic floor muscles not only during a cough but for any daily task that results in increased intra-abdominal pressure, for example during heavy lifting, Chronic coughing and heavy lifting have been cited as risk factors in the aetiology of POP so it is logical to find a way to help women with POP to counteract intraabdominal pressure increases. Thus, it is possible to apply this principle of the Knack, or pre-contracting the pelvic floor muscles, with any rise in intra-abdominal pressure due to activities such as coughing, sneezing and lifting, to prevent descent of the bladder neck and possibly other pelvic organ structures for the treatment of POP.

The aim of a PFMT treatment programme (see box 2) is to improve functional strength, co-ordination and endurance of the pelvic floor muscles. 


\section{Box 2. Aims of PFMT for prolapse}

- To improve pelvic floor muscle strength in order to improve structural support for the pelvic organs.

- To improve pelvic floor muscle coordination and timing, to provide pelvic organ support during increases in intraabdominal pressure.

\section{Content and delivery of PFMT for prolapse}

A programme of supervised PFMT includes assessment of the woman's pelvic floor muscles and her ability to contract these muscles; education about the pelvic floor muscles and how they support the pelvic organs; instruction in how to perform pelvic floor muscle exercises and the Knack. An individualised exercise programme is prescribed for the woman to follow. Adjuncts (such as biofeedback) or other physical therapies (such as neuromuscular electrical stimulation) may be used in a similar way as they would for a women receiving PFMT for urinary incontinence. Neuromuscular electrical stimulation can be used to help initiate a muscle contraction, begin muscle hypertrophy and teach the exercise technique. Biofeedback can also be used as a teaching technique to illustrate correct muscle contractions.

Currently the promotion of PFMT for prolapse varies between treatment centres with some providing only a patient information leaflet and others giving individual instruction from a physiotherapist. Research shows that verbal teaching of pelvic 
floor exercises alone is insufficient. It is suggested that $15 \%$ of women are incorrectly 'bearing down' when trying to carry out these exercises. In women with prolapse, this could further add to the strain on the area and worsen the condition. Thus ideally PFMT should be delivered by a specialist in pelvic floor rehabilitation, for example a pelvic floor physiotherapist, who will see a woman for a series of appointments in order to assess, teach and monitor progress. Many centres have such posts within obstetrics and gynaecology departments or continence services. Women are usually referred to these specialists by their GP or gynaecologist, although self-referral services are now being piloted.

Pelvic floor muscle training is considered most often for women with stage I or II prolapse, and some authors have recommended that supervised PFMT should be used for all suitable patients as a first-line treatment, much in the same way as it is for urinary incontinence where there strong evidence of its effectiveness. For women with prolapse beyond the hymen it is thought PFMT may be less effective.

A woman's motivation to adhere to a programme of PFMT is key factor to consider when recommending treatment. Successful PFMT involves a change in behaviour for the woman in order to incorporate exercises and approaches into her daily life. If a woman is not motivated to adhere the effectiveness of PFMT will be limited. Thus a thorough discussion of the woman's preferences and lifestyle is required, along with an explanation of the long-term commitment to exercise required.

\section{Effectiveness of PFMT for prolapse}


Hagen and Stark recently updated a Cochrane review which highlighted the growing evidence in support of the effectiveness of PFMT for prolapse. Currently the most rigorous evidence comes from two full-sized trials of PFMT for treatment of prolapse described below. The first by Braekken and colleagues was published in 2011 (ClinicalTrials.gov Identifier NCT00271297). The second by Hagen and colleagues was completed in April 2011 and has at this stage only been presented in a conference abstract (ClinicalTrials.gov Identifier NCT00476892).

Braekken et al undertook a single-centre trial where they randomised 109 women (59 PFMT, 50 control). Women had stage I, II or III prolapse of any type (determined by the POP-Q method of assessing prolapse severity), and $63 \%$ reported symptoms of prolapse. The intervention group women were instructed in PFMT for 6 months (weekly physiotherapy appointments for three months then fortnightly appointments for three months) with home exercise (3 sets of 8-12 close to maximal contractions daily). Both groups were given lifestyle advice and taught "the Knack". Prolapse stage, prolapse, bladder and bowel symptoms, pelvic floor muscle manometry and ultrasound measurements (blinded) were taken at 6 months.

In those women who had prolapse symptoms at baseline (69/109), those in the PFMT group compared to the control group were significantly more likely to have reduced frequency ( $74 \%$ vs $31 \%)$ and reduced bother $(67 \%$ vs $42 \%)$ with these symptoms. Fewer women in the PFMT group than the control group had no improvement in POP-Q stage (81\% versus $92 \%$ ). However, the authors reported no significant difference in the subgroup of women with prolapse beyond the hymen (80\% versus $80 \%)$. Ultrasound was used to measure the position of the bladder and 
rectum within the pelvis, and the dimensions of the muscles and hiatal area, to indicate the severity of prolapse. The change in resting position in standing of the bladder and rectum, compared to baseline, were both significantly more improved in the intervention group than the control group; and changes in measures of the muscles and hiatal area were also significantly in favour of the intervention group. Manometry was used to measure contraction strength and endurance although the assessor was not blinded to women's trial group. Braekken found the improvement in pelvic floor muscle strength and endurance were significantly greater in the intervention group compared to the control group. Better urinary outcomes for women in the intervention group were reported also.

Hagen et al conducted a parallel group, multicentre randomised controlled trial in 447 women with stage I, II or III prolapse. Women in the intervention group $(n=225)$ were randomised to PFMT, delivered by a physiotherapist at 5 appointments over 16 weeks, and lifestyle advice. Home exercise was recommended as at least three sets of exercises per day, a set consisting of up to 10 maximum voluntary contractions held for up to 10 seconds, and 10 or more fast contractions in a row. Women in the control group $(n=222)$ received a Lifestyle Advice Sheet by post only. Randomisation was by computer allocation using a remote randomisation service. Women had their prolapse assessed by a gynaecologist (POP-Q system) at baseline and (blinded) 6 months, and completed postal questionnaires at baseline (prior to randomisation) and 6 and 12 months. A 24 month follow-up is underway. The primary outcome was prolapse symptom severity (Pelvic Organ Prolapse Symptom Score - POP-SS) at 12 months. Other key outcomes were prolapse severity (POP- 
Q), women's perceived change in prolapse, uptake of further treatment and costeffectiveness.

The trial group characteristics were well-balanced at baseline. Compliance with the intervention was high: $80 \%$ of women attended 4 or 5 physiotherapy sessions. The intervention was found to be effective: compared to the control group, women's prolapse symptoms were less frequent in the PFMT group at 6 and 12 months, they were more likely to report their prolapse felt better, and less likely to seek further treatment. There was a tendency for more improvement in POP-Q stage in the intervention group, although this was only marginally significant. The cost of the PFMT intervention adjusted for further treatment received or avoided was $£ 127$ per woman. Further analysis of subgroups is planned.

These two rigorously conducted trials provide good evidence that PFMT can improve the symptoms of prolapse and the anatomical defects. Two other small trials and a large but poorly poorly-reported trial in elderly Thai women have also reported positive effects of PFMT for prolapse. It is therefore recommended to offer women with prolapse PFMT as a first option to treat their symptoms.

A few small trials have looked at PFMT as an adjunct to surgery but more evidence is needed here in order to conclude whether this is beneficial in improving surgical outcome or preventing or delaying recurrence of prolapse.

\section{Pessaries}


Pessaries offer a safe, simple and inexpensive non-surgical option for management of prolapse. The concept of pessaries for the treatment of prolapse is not a new one. Pessary use dates back thousands of years, prior to the days of Hippocrates, and innumerable varieties such as half a pomegranate, a linen tampon soaked with astringent vinegar or a piece of beef have been used over the last 200 years. It was only in the sixteenth century that a device was made specifically to be used as a pessary, as opposed to using naturally occurring objects. Since the twentieth century, considerable refinements have been made to existing pessaries. Modern pessaries are made from a variety of materials including rubber, clear plastic, soft plastic with metal reinforcements and silicone.

A range of vaginal pessaries (Figure 1) exist which can be broadly divided into two types: support (e.g. Ring, Gehrung) and space-filling pessaries (e.g. Gellhorn, Cube Donut, Inflatoball). Support pessaries lie along the vaginal axis, with the posterior component sitting in the posterior fornix and the anterior component coming to rest just under the symphysis pubis, thus providing a supportive shelf for the descending pelvic organs. A randomised trial comparing a ring with support and a Gellhorn pessary for the treatment of prolapse demonstrated no difference between the two in symptom relief reported by women.

The aim of mechanical treatment in the management of POP is to prevent worsening of the prolapse, decrease the frequency and severity of prolapse symptoms and to avert or delay the need for surgery. Support and repositioning of prolapse of pelvic organs is the commonest indication for vaginal pessary usage, but there are other less common indications (see box 3 ).

\section{Box 3. Indications for pessary use}

- Support and repositioning of prolapsed pelvic organs

- Treatment for vaginal wind 


\section{Patient assessment and pessary insertion}

The cardinal point of patient assessment is the suitability of the particular patient for the particular pessary, taking into account the sexual activity, type and degree of prolapse, ability of the patient to self-manage or attend follow-up appointments. One of the major factors to consider is the ability of the patient to attend for follow-up examinations, alone or with a carer.

At the initial visit it is recommended that the patient be examined in the recumbent as well as the standing position, during relaxation and straining. A bimanual examination is necessary in order to determine the size and type of pessary required. At this time, where possible a POP-Q examination using a speculum and Valsalva manoeuvre should be undertaken in order to demonstrate the full extent of the prolapse.

After insertion, expulsion should be checked for on movement, squatting, and carrying out the Valsalva manoeuvre. The size of the pessary should be such that it should allow a single examining finger to be passed freely all around the circumference and should not be expelled on squatting or the Valsalva manoeuvre. 
The correct pessary size and type is arrived at usually by trial and error and adequate pathways for close monitoring and review must be in place.

\section{Pessary maintenance and follow-up}

There is no consensus on the follow-up regimen as is can vary widely depending on the patient's ability to remove and self-insert the pessary, the integrity of the vaginal epithelium and complications. The patient must be informed of the symptoms of potential complications and should be advised to be aware of any change in her voiding pattern. Although there is no consensus on the duration, periodic vaginal inspections are recommended. Any frank ulceration or vaginal excoriation should prompt discontinuation of the pessary until the vaginal skin heals. Refitting with a smaller sized pessary or a different shaped pessary should then be considered. Mild vaginal irritation is common and does not necessitate discontinuation of the pessary. Although there is no consensus, six monthly follow-up of self-inserting patients is recommended with more frequent follow-up visits for patients who are unable to selfinsert. Effective service can been provided by all levels of health professionals from hospital specialists, general practitioners and specialist nurses.

\section{Effectiveness of pessary usage}

Pessaries have been shown to be largely successful for the remission of most of the symptoms attributable to POP i.e. prolapse, bladder and bowel symptoms. There is no agreement in the literature on what is considered successful fitting of a pessary. Some authors have considered fitting successful if a pessary was perceived comfortable by a patient when retained during Valsalva and voiding at the initial visit, while others considered it successful if a patient continued to use the pessary until 
the following visit to the doctor. Therefore, the rates of successful fitting vary between $89 \%$ to $8 \%$, with the follow-up time to define success ranging from initial fit to 7 years. A Cochrane review by Adams identified a lack of rigorous evidence for the effectiveness of pessaries and their optimal management. Women who have had several pregnancies, a previous hysterectomy or a previous prolapse repair often have difficulty retaining a pessary, which may be due to increased laxity, perineal incompetence or narrowing of the vaginal passageway after surgery.

\section{Complications}

Common complications are bleeding, vaginal excoriations, ulcerations and impactions in the vagina, while rare complications include actinomycosis and bacterial vaginosis. Vaginal ulcerations and excoriations can be treated effectively by vaginal oestrogen application. A woman with a neglected pessary can present with more serious complications namely fistula formation and peritonitis. Erosion into the bowel or bladder and dense adhesions to other pelvic structures have been reported. Unusual complications of cervical entrapment, small bowel incarceration, and hydronephrosis, vaginal cancer have also been reported. Complications can be minimised by regular change and follow-up care of the pessary.

\section{Figure 1: Various types of pessaries}




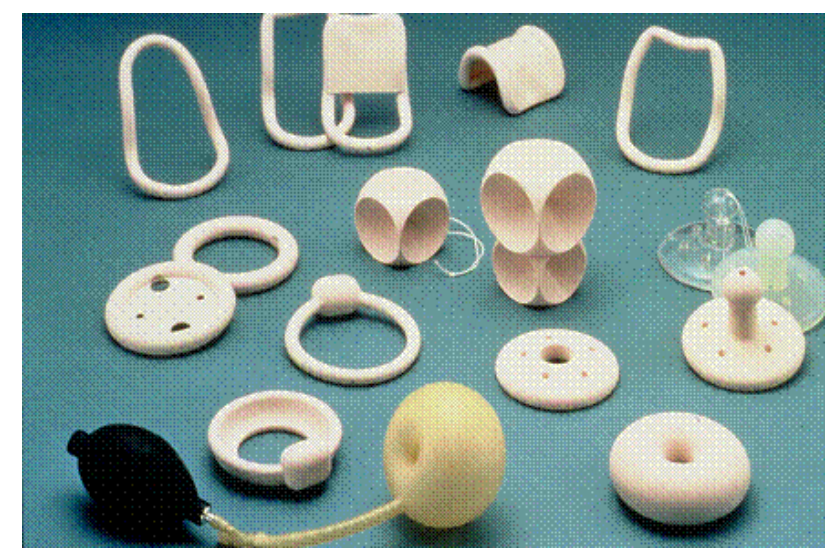

Photograph provided by Mediplus

\section{Practice Points.}

- Lifestyle changes such as weight loss, avoiding heavy lifting and avoiding constipation may have a positive impact on prolapse symptoms and should be recommended.

- A one to one pelvic floor muscle training programme can reduce severity and symptoms of prolapse for women with stage I to III prolapse.

- Vaginal pessaries are an effective and acceptable option for some women with prolapse. There is a lack of evidence regarding which types of pessary are best for different presentations, and the optimal follow-up regime.

\section{Further reading}

Hagen S, Stark D. Conservative prevention and management of pelvic organ prolapse in women. Cochrane Database of Systematic Reviews 2011, Issue 12. Art. No.: CD003882. DOI: 10.1002/14651858.CD003882.pub4. 
Adams E, Thomson A, Maher C, Hagen S. Mechanical devices for the management of pelvic organ prolapse in women. The Cochrane Library/Cochrane Incontinence Group 2004

\section{Conflict of interest statement}

Suzanne Hagen - no conflicts to declare

Ranee Thakar - no conflicts to declare 